\title{
VELOCITY VECTOR CONTROL OF A LINEAR PERMANENT MAGNET SYNCHRONOUS MOTOR
}

The paper presents a design of vector control algorithm for electric drive employing a linear permanent magnet synchronous motor (LPMSM). For simulation and further experimental verification it was necessary to formulate a mathematical model of the LPMSM. This model was derived from a standard mathematical model of a rotary PMSM and then exploited to verify vector control of the machine. The presented simulation results are used to control the LPMSM drive system with maximum efficiency.

\section{Introduction}

The construction principles of linear motors are known as long as principles of rotational motors. The principle of the induction linear motor was described by Charles Wheatson in 1841, [1]. But these motors have been exploited in industrial applications for last ten years mainly. This expansion was enabled by the development of new materials for permanent magnets, new controlled elements and topologies of power converters and new control techniques, which are suitable for applications with linear motors.

\section{Basic Principles of Linear Motors}

There are two basic definitions of linear motors. One of them introduces linear motors as a classical synchronous or induction rotational motor, where the transformation from rotary to linear system is shown in Fig. 1. The second definition describes generally a linear motor as a corresponding rotational motor with the infinite stator and rotor radius.

The stator of linear motors is usually called primary part and the rotor is called secondary part. The primary part consists of ferromagnetic plates and three phase winding, which is stored in slots. Opposite of the primary part is secondary part, sometimes called mover, which contains permanent magnets, usually made from Neodymium - Iron - Boron (Ne-Fe-B). Magnets are stacked on the iron base. In induction linear motors, the secondary part is composed as a squirrel cage stored in slots, or stacked on the iron base or sometimes as a simple aluminium sheet. For many applications it is a primary part which is moved on the path as a mover. The path is usually composed of many sections of permanent magnets, which create a secondary part. Such concept requires flexible supply cables, flexible position sensor cable and for high power linear motors also flexible tubes for water cooling.

In general, linear drives consist of a linear motor, power converter and position or velocity sensors. For industrial applications the linear drives also need special equipment such as a guiding system, extra cooler and safety parts. Due to the fact that many linear drives are position controlled servo drives, these applications need for control a special circuitry which usually utilises the information from a position or velocity sensor.

\section{Principles of Vector Control of LPMSM}

Vector control is used the most often for control of electric drives for which a high precision and fast response to the demanded values of speed or position are required. This is impor-

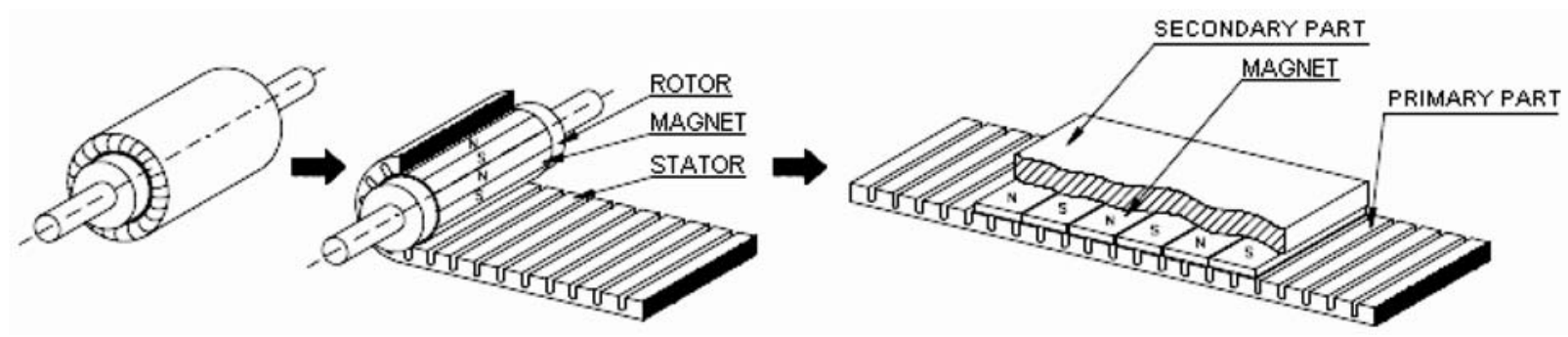

Fig. 1 Analogy between rotary motor and linear motor

\footnotetext{
* Vladimir Vavrus, Jan Vittek, Michal Malek

Department of Power Electrical Systems, Faculty of Electrical Engineering, University of Zilina, Slovakia, E-mail: Vladimir.Vavrus@kves.uniza.sk,
} 
tant for steady-states as well as for transient states of the drive. The basic idea is to achieve control properties of the drive with a separately-excited dc motor. For dc motors positions of magnetic flux vector (created by stator winding) and armature current are mutually orthogonal (given by commutator brushes position) to create always maximum torque of the machine. Due to the fact that exciting winding and armature winding are not only separately mounted inside of the motor but also separately supplied, these two control variables can be controlled independently. Exploiting this analogy, the basic principle of vector control of the LPMSM is independent control of two state variables, currents of primary part and magnetic flux [2]. The most widely used block diagram for velocity control of the LPMSM has a cascade structure, which is shown in Fig. 2.

$$
F=\frac{3}{2} \cdot K_{x} \cdot \Psi_{P M} \cdot i_{q} F=\frac{3}{2} \cdot K_{x} \cdot \Psi_{P M} \cdot\left|I_{s}\right| \cdot \sin \alpha_{m}(2 \mathrm{a}, \mathrm{b})
$$

where: $\alpha_{m}$ is the angle between vectors of primary part $I_{s}$ current and permanent magnet flux $\Psi_{P M}$.

During such conditions the flux of permanent magnet $\Psi_{P M}$ is maintained constant and maximal force is achieved for angle $\alpha_{m}=90^{\circ}$. This condition is satisfied for $d-q$ coordinate system, which has $d$-axes identical with the direction of the permanent magnet flux and a primary part current vector in this frame is orthogonal to $d$-axes and therefore $i_{d}$ current will be 0 .

The structure of velocity vector control of the LPMSM is shown in Fig. 2. It has a nested structure which consists of two loops.

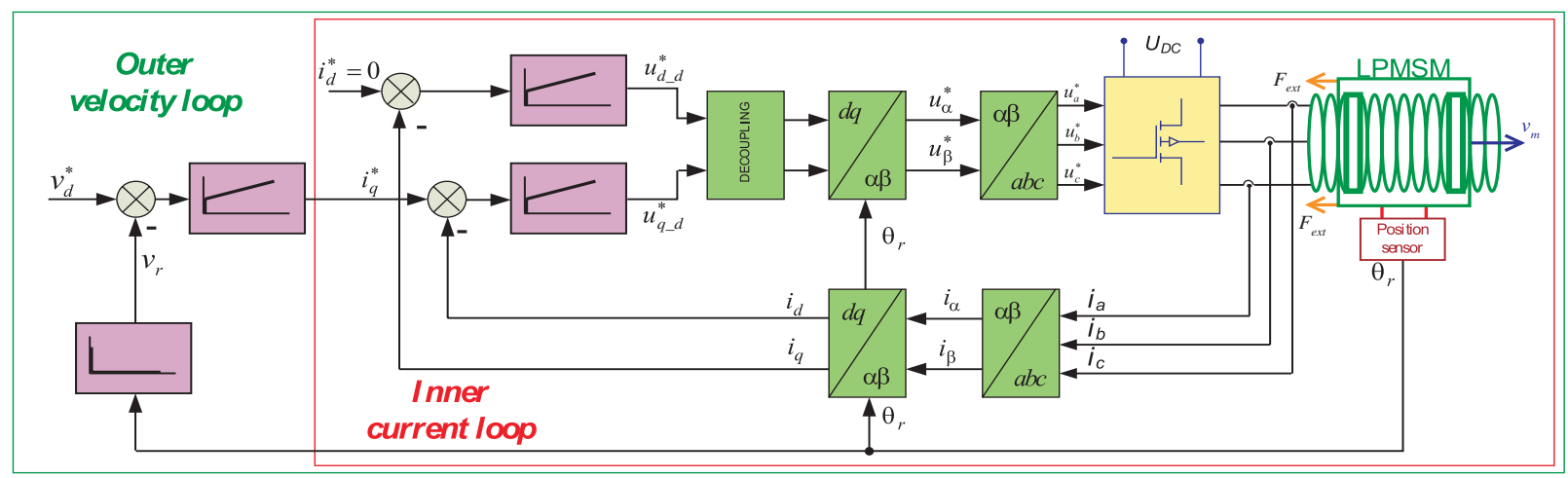

Fig.2 Typical cascade structure for velocity vector control of LPMSM

\subsection{Vector control applied to LPMSM}

The basic principle of vector control strategy for the LPMSM is decomposition of a primary part phase current vector into two orthogonal components. The first component is $i_{d}$ current component (which produces a magnetizing flux). This component is in the phase with permanent magnet flux. The second component is $i_{q}$ current (which produces an electromagnetic force), which is orthogonal to the first one (see Fig. 3). For rotor flux-oriented vector control of the LPMSM, the direct-axis stator current and the quadrature-axis stator current must be controlled independently. The force produced by the motor is in a $d-q$ coordinate frame equal (details in sect. 4.1):

$$
F=\frac{3}{2} \cdot K_{x} \cdot\left[\Psi_{P M} \cdot i_{q}+\left(L_{d}-L_{q}\right) \cdot i_{d} \cdot i_{q}\right]
$$

Equation (1) describes two parts of the motor force. First one 'magnetic' is independent of the primary part current $i_{d}$, and it is proportional to $i_{q}$ current. The second part 'reluctance' is proportional to both currents $i_{d}, i_{q}$ of the primary part multiplied by the difference between primary part inductances in $d-q$ axes $\left(L_{d}-L_{q}\right)$.

When the direct a quadrature inductances are the same, eq. (1) can be simplified as (2a), from which the most efficient control by controlling $i_{q}$ current only, can be determined as (2b):

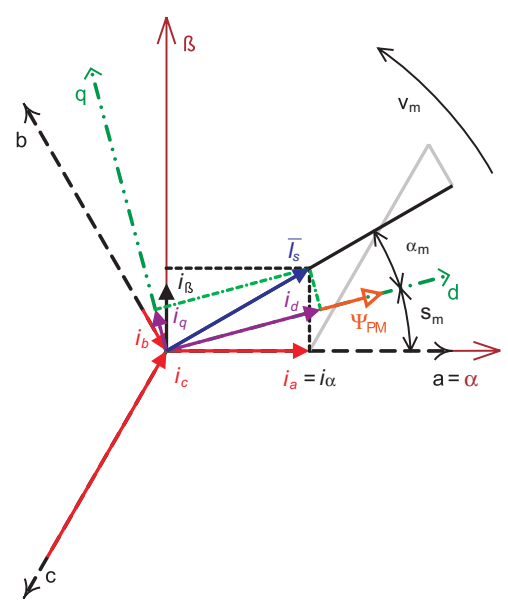

Fig. 3 Phase diagram of stator current

The outer loop is a velocity control loop and the inner loop is a current control loop. The value of the demanded current $i_{q}$ (force producing component) is determined by the velocity PI regulator having the difference between demanded velocity, $v_{d}$ and real motor velocity, $v_{m}$ as an input. Controlling $i_{d}$ current at 0 value, the motor will produce maximal electromagnetic force up to the nominal 

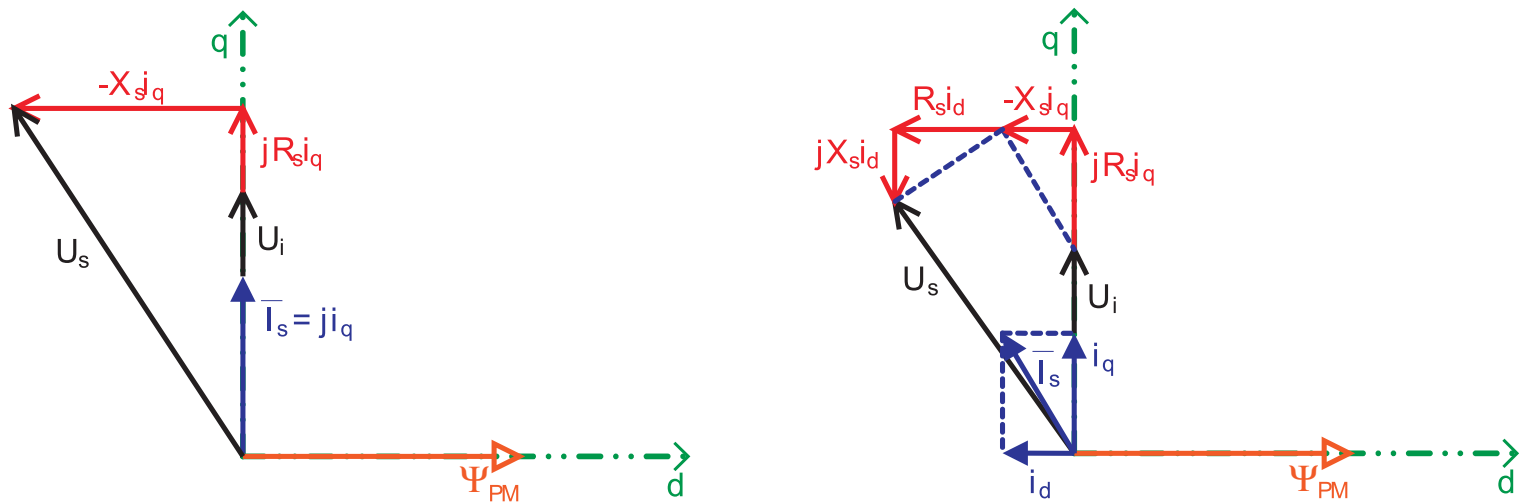

Fig. 4 Phase diagram of LPMSM vector control

a) up to nominal velocity, $b$ ) over a nominal velocity

velocity. The phase diagram shown in Fig. 4a describes normal vector control up to the nominal velocity. If a higher velocity than nominal is demanded then the field weakening must be applied by controlling the $i_{d}$ current, which is shown as the phase diagram in Fig. $4 b$.

\section{Simulation of LPMSM Vector Control}

Verification of the designed LPMSM vector control was made in Matlab-Simulink environment. The block diagram of the proposed control is shown in Fig. 5. Simulations run with different sampling frequencies for the both control loops and the model of the motor. Sampling frequency of the outer velocity control loop is $1 \mathrm{kHz}$ and inner current control loop has the sampling frequency of $10 \mathrm{kHz}$. The LPMSM model in orthogonal $d-q$ transformations has a sampling frequency of $1 \mathrm{MHz}$.

However, the equations for primary part voltage components are coupled. The demanded voltages are increased for the part which contains also decoupling components as follows:

$$
u_{d}=u_{d_{-} d}-L_{q} \cdot i_{q} \cdot v_{m} \quad \text { and }
$$

$$
u_{q}=u_{q_{-} d}+L_{d} \cdot i_{d} \cdot v_{m}+K_{e} \cdot v_{m},
$$

where: $u_{d_{-} d}, u_{q_{-} d}$, are the demanded voltages from current PI regulators.

The parameters of the primary part of the real LPMSM which was built in DPES are listed in Tab. 1 and used for simulations. The real LPMSM has four sections of the primary part which are connected in series.

Parameters of one primary part of LPMSM Table 1

\begin{tabular}{|c|c|c|c|c|c|c|c|}
\hline$R_{s}[\Omega]$ & 2.35 & $L_{q}[\mathrm{mH}]$ & 0.12 & $\tau_{p}[\mathrm{~mm}]$ & 82.5 & $K_{e}\left[\mathrm{Vsm}^{-1}\right]$ & 52.9 \\
\hline$L_{d}[\mathrm{mH}]$ & 0.12 & $\mathrm{p}[-]$ & 4 & $K_{f}\left[\mathrm{NA}^{-1}\right]$ & 45.8 & $M[\mathrm{~kg}]$ & 40 \\
\hline
\end{tabular}

\subsection{LPMSM mathematical model}

The LPMSM mathematical model can be derived through analogy to a rotary permanent magnet synchronous motor (PMSM). After transformation from the tree-phase $a-b-c$ frame to the equiv-

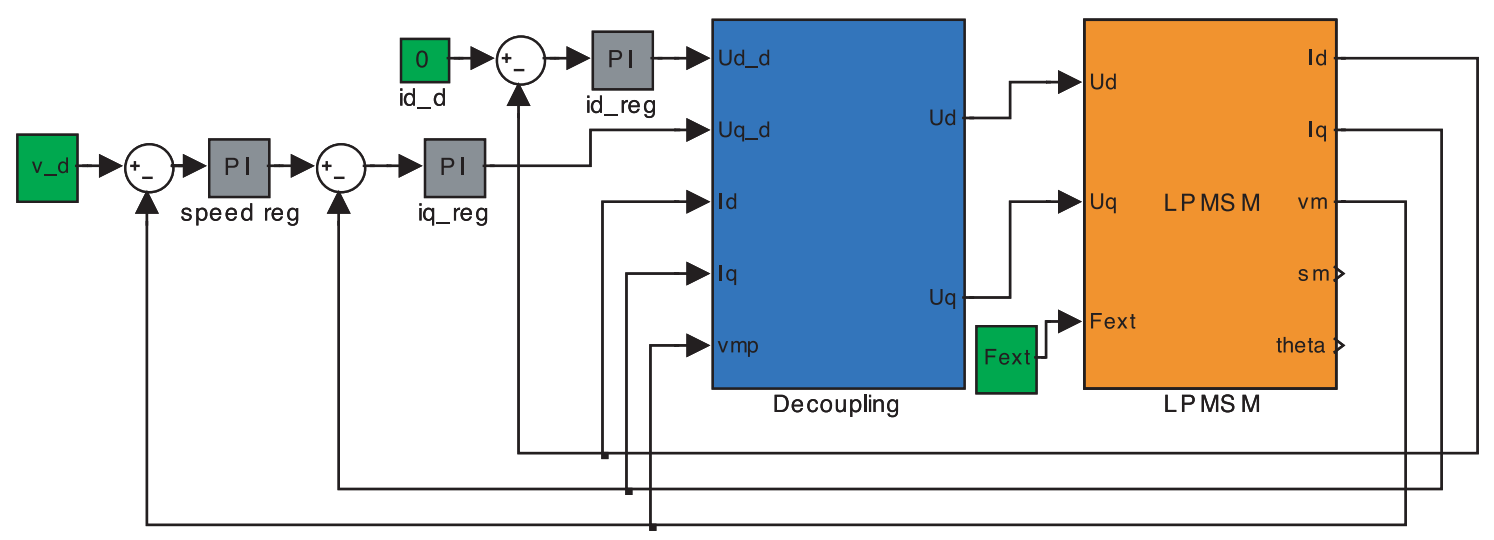

Fig. 5 Simulation model of vector control of PMLSM 
alent orthogonal synchronous $d-q$ frame, the relations between the $d-q$ components are given as [1], [3] and [4]:

$$
\begin{aligned}
& u_{d}=R_{s} \cdot i_{d}+\frac{d \psi_{d}}{d t}-\omega_{e} \cdot \psi_{q}, \\
& u_{q}=R_{s} \cdot i_{q}+\frac{d \psi_{q}}{d t}-\omega_{e} \cdot \psi_{d}, \\
& \psi_{d}=L_{d} \cdot i_{d}+\Psi_{P M}, \\
& \psi_{q}=L_{q} \cdot i_{q} \\
& J \frac{d \omega_{e}}{d t}=\frac{3}{2} \cdot p \cdot\left(\psi_{d} \cdot i_{q}-\psi_{q} \cdot i_{d}\right)-\Gamma_{e x t}, \\
& \omega_{e}=p \cdot \omega_{r},
\end{aligned}
$$

where:

$R_{s} \quad$ is the phase winding resistance,

$\omega_{r} \quad$ is the mechanical angular speed,

$p \quad$ is the number of pole-pairs,

$J \quad$ is the lumped moment of inertia,

$\Gamma_{e x t}$ is the external load torque,

$u_{d}, u_{q}$ are the $d$ and $q$ axis voltages,

$i_{d}, i_{q}$ are the $d$ and $q$ axis currents,

$\psi_{d}, \psi_{\mathrm{q}}$ are the $d$ and $q$ axis fluxes,

$\Psi_{P M}$ is the permanent magnet flux linkage,

$\omega_{e} \quad$ is the electrical angular speed.

Equations (4) - (7) represent voltages respective fluxes equations of the rotary PMSM in the $d-q$ coordinate system, where $L_{d}$, $L_{q}$ are the $d$ and $q$ axis inductances. Eq. (8) is an electro-mechanical equation of the PMSM and eq. (9) defines the relation between electrical and mechanical angular speed.

Linear motors, unlike rotary motors, produce a translation movement, therefore, it is necessary to define the rate constant between the stationary reference frame and the rotating reference frame. This definition is valid only when the synchronous angular speed is the same as the electrical angular speed $\omega_{s}=\omega_{e}$.

$$
K_{x}=\frac{\omega_{e}}{v_{m}}=\frac{2 \cdot \pi \cdot f_{s}}{v_{m}} \text { and } v_{m}=2 \cdot \tau_{p} \cdot f_{s}
$$

where:

$v_{m} \quad$ is the electric linear velocity,

$\tau_{p} \quad$ is the pole pitch

$f_{s} \quad$ is the frequency of the power supply voltage

$K_{x} \quad$ is the rate constant between the stationary reference frame and the linear reference frame

Using eq. (4) - (8) together with eq. (10a,b) a new form of stator voltage equations can be derived:

$$
\begin{aligned}
& u_{d}=R_{s} \cdot i_{d}+L_{d} \frac{d i_{d}}{d t}-K_{x} \cdot L_{q} \cdot i_{q} \cdot v_{m}, \\
& u_{q}=R_{s} \cdot i_{q}+L_{q} \frac{d i_{q}}{d t}+K_{x} \cdot L_{d} \cdot i_{d} \cdot v_{m}+K_{x} \cdot \Psi_{P M} \cdot v_{m},(12)
\end{aligned}
$$

If the mechanical power of the rotary and linear motor are same, then it is possible to derive:

$$
M_{m} \cdot \omega_{r}=F_{m} \cdot v_{m} \Rightarrow M_{m}=F_{m} \cdot \frac{v_{m}}{\omega_{r}}=F_{m} \cdot \frac{\tau_{p}}{\pi} \cdot p,
$$

where:

$M_{m} \quad$ is the mechanical torque of the rotational PMSM

$F_{m} \quad$ is the mechanical trust of the linear PMSM

When the principles of vector control are respected then electromagnetic torque of the rotational PMSM is done as:

$$
M_{m}=\frac{3}{2} \cdot p \cdot \Psi_{P M} \cdot i_{q} .
$$

Comparing (13) and (14) the electromechanical force of the LPMSM can be expressed as:

$$
F_{m}=\frac{3}{2} \cdot \frac{\pi}{\tau_{p}} \cdot \Psi_{P M} \cdot i_{q}=\frac{3}{2} \cdot K_{x} \cdot \Psi_{P M} \cdot i_{q}=K_{f} \cdot i_{q},
$$

where: $K_{f}$ is the force constant of the LPMSM.

The definition of the voltage constant $K_{e}$ is as follows:

$$
K_{e}=\frac{\pi}{\tau_{p}} \cdot \Psi_{P M}=K_{x} \cdot \Psi_{P M} .
$$

Applying the constant (16) into the equations (11) and (12) the new stator voltage equations are defined as:

$$
\begin{aligned}
& u_{d}=R_{s} \cdot i_{d}+L_{d} \frac{d i_{d}}{d t}-K_{x} \cdot L_{q} \cdot i_{q} \cdot v_{m}, \\
& u_{q}=R_{s} \cdot i_{q}+L_{q} \frac{d i_{q}}{d t}-K_{x} \cdot L_{d} \cdot i_{d} \cdot v_{m}+K_{e} \cdot v_{m} .
\end{aligned}
$$

Using the force constant and a vector control strategy (the demanded current in d axis up to nominal velocity is zero, $i_{d_{-} d}=0$ ) a mechanical equation for the LPMSM has the form:

$$
\begin{aligned}
& \frac{d v_{m}}{d t}=\frac{1}{M}\left(F_{m}-F_{e x t}\right)=\frac{1}{M}\left(K_{f} \cdot i_{q}-F_{e x t}\right) \text { and } \\
& \frac{d s_{m}}{d t}=K_{x} \cdot v_{m}
\end{aligned}
$$

where: $M$ is the total mass of the moving element system,

$F_{\text {ext }}$ is the external disturbance term (external load force), $s_{m}$ is the position of moving element.

The mathematical model of the LPMSM in Matlab-Simulink environment is shown in Fig. 6 .

\subsection{Simulation Results}

The simulation results of the LPMSM vector control are summarised in Fig. 7. Fig. 7a shows demanded velocity, real motor velocity and applied external load force as a function of time and Fig. $7 \mathrm{~b}$ presents the motor currents in a synchronously moving reference frame in $d-q$ axes as a function of time. 


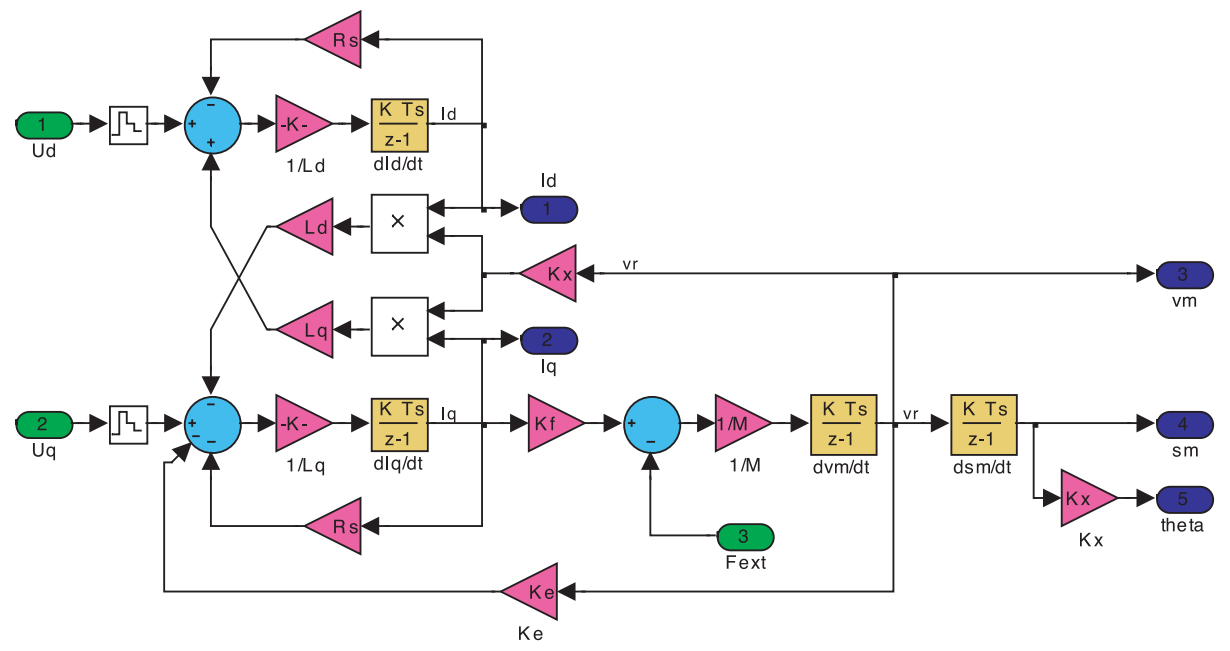

Fig. 6 Matlab-Simulink model of PMLSM.
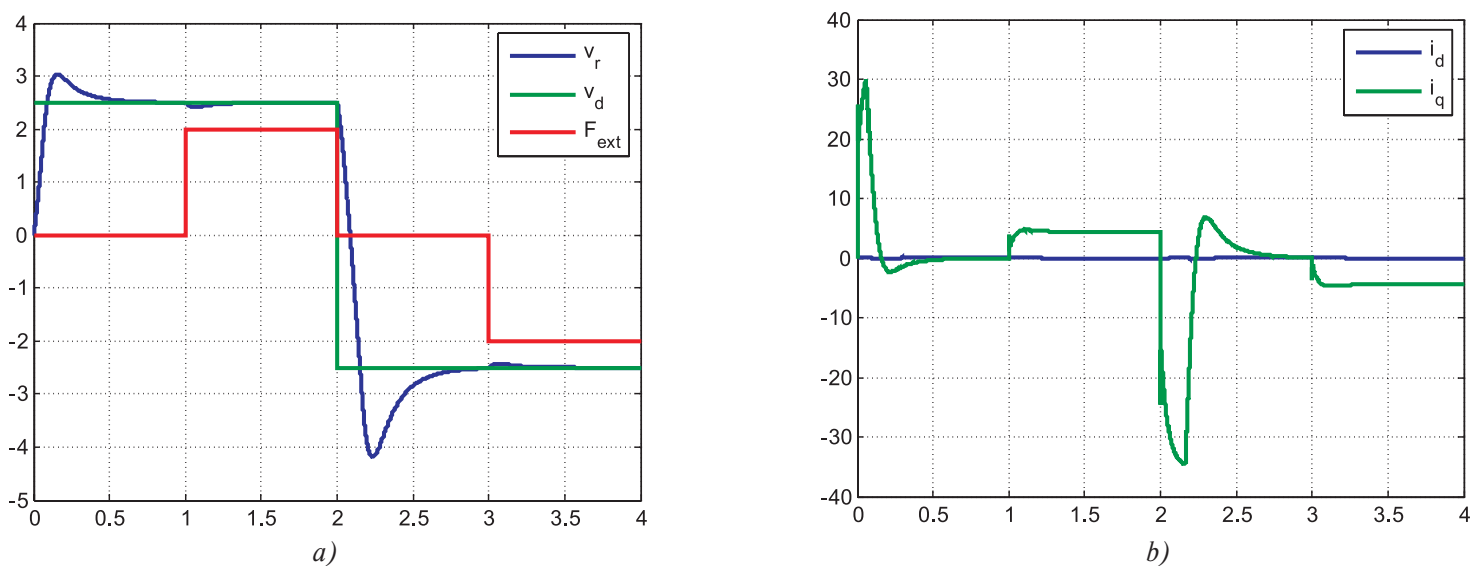

Fig. 7 Simulation results, a) motor velocity and applied external load force vs. time; b) motor current components vs. time in d-q reference frame

The simulation results of the proposed vector control for electric drives employing the LPMSM show a good agreement with theoretical predictions. As can be seen from Fig. 7a the LPMSM reach the demanded velocity in both directions of turning with a small overshoot. Fig. $7 \mathrm{~b}$ demonstrates the proper operation of both regulators. The flux regulator keeps $i_{d}$ current component at the zero value as it is demanded up to the nominal velocity of the motor and the force regulator produces $i_{q}$ current component to reach the demanded velocity for both directions and also to cover the external force disturbance.

\section{Conclusion}

Based on the analogy between a permanent magnet synchronous motor and the LPMSM this paper developed a mathematical model of the machine. The model is then exploited to derive vector control of the drive with the LPMSM. Vector control of the rotational and linear motor is based on the same principles, but it is important to precisely define the differences between them. Analogy of both motors enabled that all the fundamental equations of the LPMSM where derived in an orthogonal synchronous $d-q$ frame, and from them the LPMSM model Matlab-Simulink environment was built and tested for a designed vector control strategy. The presented simulation results predicted possibility to control this motor exploiting vector control technique and also confirmed the possibility to achieve good drive performance.

Preliminary experimental investigation of the proposed control technique of the LPMSM already started and experimental results on the proposed drive system with the LPMSM would be a natural next step. Extension of the vector control algorithm to the forced dynamics control would be of interest.

\section{Acknowledgements}

The authors wish to thank Slovak Grant Agency VEGA for funding the project No.4087/07 'Servosystems with Rotational and Linear Motors without Position Sensor'. 


\section{References}

[1] ZALMAN, M., JOVANKOVIC, J.: New trends in control of linear motors (in Slovak), AT\&P journal 2/2006, pp. 67-70.

[2] BOLDEA, I., NASAR, S. A.: Vector Control of AC Drives, CRC Press, Boca Raton, 1992

[3] VITTEK, J., VAVRUS, V., MALEK, M., BUCHNER, P., MICHALIK, W.: Prescribed Closed-loop Speed Dynamics Control of the Actuator employing Linear Permanent Magnet Synchronous Motor, ICIT 05, Hong-Kong.

[4] ZENG, J., BARRE, P., DEGOBERT, P.: Modeling and Trust Control of PMLSM Using Principle of Local Energy, ICEMS 2003, Vol. 1, 2003, pp. 26-30.

[5] HONG, G., KAIPEI, L.: Model of the permanent magnet linear synchronous motor on the bias of the field orientation, Electrical Machines and Systems, ICEMS 2005, Vol. 1, pp. 442-445. 